\title{
ANÁLISIS DE LOS HÁBITOS DE ESTUDIO EN UNA MUESTRA DE ALUMNOS UNIVERSITARIOS
}

\author{
Valentín Martínez-Otero Pérez y Liliana Torres Barberis \\ Centro de Enseñanza Superior "Don Bosco, España
}

\section{INTRODUCCION}

La preocupación por los hábitos de estudio de los alumnos viene de antiguo. No obstante, en nuestro tiempo esta cuestión adquiere un renovado interés, en gran medida por la extensión de la educación, así como por las altas tasas de fracaso escolar. De hecho, diversas investigaciones se orientan a conocer con exhaustividad los procesos de aprendizaje y a valorar en qué grado influyen los hábitos y técnicas de estudio en el rendimiento académico. Ahora bien, llama la atención la escasez de prospecciones de este tipo en universitarios, acaso porque se supone que cuando los estudiantes ingresan en la Universidad ya poseen unos hábitos de estudio suficientemente aceptables. Sin embargo, la experiencia nos demuestra que un número significativo de alumnos de enseñanza superior obtienen malos resultados. En efecto, no todos los estudiantes hacen frente con éxito a los nuevos desafíos que la Universidad plantea: aumento de la exigencia, necesidad creciente de organización del trabajo académico, mayor dedicación al estudio, autonomía, etcétera.

El presente trabajo parte de la idea de que el estudio, en tanto que esfuerzo intencional encaminado al aprendizaje, condiciona el rendimiento intelectual y académico de los alumnos. Por lo mismo, se exponen los resultados de una investigación realizada con universitarios de primer curso de las diplomaturas de Magisterio y de Educación Social, al tiempo que se repasan algunos relevantes aspectos relativos a la actividad de estudiar.

\section{HABITOS DE ESTUDIO}

Aunque las variables que condicionan el rendimiento académico en los distintos niveles de enseñanza son numerosas y constituyen una intrincada red en la que resulta harto complejo ponderar la influencia específica de cada una, se ha generalizado la idea de que los hábitos de estudio influyen considerablemente en los resultados escolares. Naturalmente, no se deben confundir los hábitos (prácticas constantes de las mismas actividades) con las técnicas (procedimientos o recursos). Unos y otras, empero, coadyuvan a la eficacia del estudio. De un lado, el hábito de estudiar es necesario si se quiere progresar en el aprendizaje. De otro, conviene sacar el máximo provecho a la energía que requiere la práctica consciente e intensiva del estudio por medio de unas técnicas adecuadas.

La pretensión de mejorar el estudio es una constante pedagógica. Muchas de las propuestas de optimización de esta actividad se han realizado por los propios maestros y profesores sobre la base de su experiencia e intuición, lo que ha permitido alcanzar metas nada desdeñables. Junto a estas aportaciones naturales, tradicionales y espontáneas, hay que considerar las contribuciones provenientes de la psicología conductista y cognitiva. Sin pretensión de exhaustividad, recogemos algunas de las implicaciones que se derivan para el estudio de los dos paradigmas citados (Hernández y García 1991): 
Paradigma conductista.- Se considera el estudio como una compleja secuencia de acciones: elección del tema, organización del material, lectura del texto y adquisición de contenidos. Lo que se busca es conocer las situaciones antecedentes de la conducta de estudio y utilizar refuerzos apropiados que permitan obtener rendimientos satisfactorios. Algunas de las consideraciones ofrecidas por este paradigma respecto al estudio son:

- Las condiciones del estudio deben ser apropiadas: temperatura, ventilación, mobiliario, iluminación, organización de materiales, tiempo de estudio, horarios, etc.

- Hay que dar a conocer al alumno técnicas de estudio: diferenciación entre ideas principales y secundarias, realización de esquemas y resúmenes, utilización de diccionarios, manejo de ficheros, etc.

- Estructurar la tarea de estudio, dividiéndola en sus componentes específicos y desarrollando el aprendizaje gradualmente.

- Tener en cuenta la curva de aprendizaje: precalentamiento, ascenso, meseta, descenso y fatiga.

- Incluir períodos de descanso que permitan disminuir el cansancio y potenciar el aprendizaje.

- Potenciar la motivación del alumno por el estudio.

- Utilizar refuerzos que faciliten y consoliden los hábitos y técnicas de estudio

Como puede comprobarse, desde esta perspectiva se quieren promover hábitos y técnicas de estudio que mejoren con la práctica, pero se soslayan los mecanismos internos de la adquisición y de la elaboración informativa. A este paradigma se le critica el eficientismo, la algoritmización y la limitación de la libertad del alumno al estudiar, ya que no se tiene en cuenta la intencionalidad del comportamiento.

Paradigma cognitivo. - Se centra sobre todo en el aprendizaje de contenidos y, por tanto, enfatiza la importancia de la memoria, la codificación y la recuperación informativa. Las principales aportaciones son:

- La memorización de contenidos informativos representa el proceso más característico de la conducta de estudio.

- Hay un interés especial por las estructuras y procesos cognitivos más relevantes: almacenes informativos, representaciones mentales, relación entre informaciones viejas y nuevas, leyes del olvido, etc.

- La mente es un "sistema constructor" de la información.

- La estructura del texto se corresponde con las estructuras mentales. De acuerdo con este principio, el texto es un medio extraordinario para comprender el funcionamiento de la mente humana y la actividad de estudiar.

- Para mejorar el estudio es imprescindible desarrollar ciertas operaciones cognitivas.

- Las técnicas de trabajo intelectual adquieren especial importancia en el estudio, porque permiten entrenar al alumno en determinadas estrategias de atención, elaboración y 
organización de la información, al tiempo que se favorece la metacognición o regulación de los propios procesos de pensamiento y de aprendizaje.

Así pues, este paradigma se interesa principalmente por los procesos mentales, la comprensión de textos y la metacognición. Tiene el inconveniente de desatender aspectos no cognitivos que pueden influir en el estudio y en los logros escolares.

Por nuestra parte, consideramos que los dos paradigmas mencionados nos ofrecen aportaciones relevantes para la comprensión del estudio. La complejidad de la actividad de estudiar exige tener presente las distintas contribuciones, para no caer en el error de adoptar visiones parciales y reducidas. El enlace entre las dos teorías nos ayuda a explicar el proceso de estudio, mas creemos oportuno invocar la importancia de las variables afectivas, grupales y sociales apenas contempladas por los paradigmas conductista y cognitivo. Desde un enfoque integral de la persona, como el que defendemos, es evidente que en la comprensión de la actividad de estudiar hay que prestar atención a los valores, actitudes e intereses de los educandos. En este sentido, el vacío que dejan los investigadores de la conducta y de la cognición se llena si consideramos el estudio como una parte del proceso de formación total. Esto nos lleva a afirmar que para entender el procesamiento de la información y la representación del conocimiento, al igual que las acciones más o menos ordenadas y observables que realiza el alumno para la obtención de determinados resultados, es necesario apelar a otras dimensiones: la autoestima y el autoconcepto, la motivación y los intereses, las expectativas de éxito, el clima social del aula y del centro, el ambiente familiar, la cooperación entre alumnos, etc. El estudio, pues, es una vertiente del proceso de crecimiento personal que supone el desarrollo armónico de la inteligencia, la voluntad y la creatividad.

De acuerdo a la perspectiva humanista que se adopta en este trabajo, cualquier plan de adquisición de hábitos de estudio debe partir de las necesidades, capacidades, afectos, actitudes y valores de los alumnos, sin que ello nos haga olvidar el papel de la orientación. La trascendencia de estos planteamientos, así como sus aplicaciones e implicaciones se dejan sentir particularmente en la investigación que hemos realizado. Los hábitos de estudio de alumnos universitarios de Magisterio y de Educación Social han de guardar relación con la preparación que reciben y con las relevantes funciones que están llamados a realizar en un futuro próximo como formadores. Los estudiantes de estas diplomaturas deben tener presente que de su formación dependerá en buena parte el aprendizaje de los educandos con los que trabajen.

En suma, los hábitos y estrategias de estudio deben guardar relación con el futuro perfil profesional del alumno universitario. Por ejemplo, las técnicas de trabajo del estudiante de Ingeniería difieren considerablemente de las del alumno de Filosofía. Por supuesto, el hábito de estudiar es importante en todos los casos, mas pensamos que en las carreras de Magisterio y de Educación Social adquieren especial importancia la reflexión, la creatividad y la cooperación, cualidades todas que deben reunir los buenos educadores y que difícilmente se explican desde los paradigmas conductista y cognitivo.

\section{3.- INVESTIGACION REALIZADA}

\section{1.- Muestra}

El trabajo se desarrolló a lo largo de un curso académico. La investigación se realizó desde el Servicio de Orientación del C.E.S. "Don Bosco", con el propósito de conocer los hábitos de estudio de los 
alumnos de primer curso de Magisterio y de Educación Social. La distribución de escolares de la muestra por especialidades es la siguiente:

Diplomatura de Magisterio

1.- Audición y Lenguaje

$\mathrm{N}=22$

2.- Educación Especial

$\mathrm{N}=22$

3.- Lengua Extranjera

$\mathrm{N}=12$

4.- Educación Infantil

$\mathrm{N}=29$

5.- Educación Física

$\mathrm{N}=10$

6.- Educación Primaria

$\mathrm{N}=23$

Diplomatura de Educación Social

7.- Especialidad única

$\mathrm{N}=19$

Nuestra pretensión era disponer de una muestra suficientemente amplia, homogénea y representativa del conjunto de alumnos que realizan el primer curso de la carrera en el Centro. La muestra total está compuesta por 137 alumnos.

\section{2. - Instrumento utilizado}

Se aplicó el Inventario de Hábitos de Estudio (I.H.E.) de Pozar (1989). Este instrumento se orienta a un triple objetivo:

- Diagnosticar la naturaleza y grado de los hábitos de estudio de los alumnos.

- Pronosticar la incidencia de los hábitos de estudio en el aprendizaje.

- Actuar sobre los hábitos de estudio de los alumnos, de manera tal que se corrijan los inadecuados y se adquieran y consoliden los apropiados.

El I.H.E. mide los hábitos de estudio de los alumnos por medio de cuatro escalas fundamentales, a las que se añade una escala de sinceridad:

Escala I: Condiciones ambientales del estudio:

1.- Condiciones ambientales personales

2.- Condiciones ambientales físicas 
3.- Comportamiento académico

4.- Rendimiento

Escala II: Planificación del estudio:

5.- Horarios

6.- Organización

Escala III: Utilización de materiales

7.- Manejo de libros

8.- Lectura

9.- Subrayado-resúmenes

Escala IV: Asimilación de contenidos

10.- Memorización

11.- Personalización

Escala V: Sinceridad (adicional)

\section{3. - Resultados}

Se presentan a continuación de forma gráfica los perfiles correspondientes a las distintas especialidades de Magisterio y de Educación Social, así como el perfil de toda la muestra. 
Especialidad: Audición y Lenguaje

Número de alumnes : 22

P E R F I L

\begin{tabular}{|c|c|c|c|c|c|c|c|c|c|c|}
\hline \multirow{2}{*}{ Escala } & \multirow{2}{*}{ P. D. } & \multirow{2}{*}{$\begin{array}{c}\text { Ma] } \\
1\end{array}$} & \multicolumn{2}{|c|}{ No sutisfinctorio } & \multicolumn{3}{|c|}{ Normal } & \multicolumn{2}{|c|}{ Bien } & \multirow{2}{*}{$\begin{array}{c}\text { Excelente } \\
9\end{array}$} \\
\hline & & & 2 & 3 & 4 & 5 & 6 & 7 & 8 & \\
\hline I & 23 & 0 & 0 & 0 & $\circ$ & $\circ$ & $\stackrel{0}{\circ}$ & $\longrightarrow$ & $\%$ & 0 \\
\hline II & 9 & 0 & & $\pi$ & $\circ$ & 。 & 0 & 0 & 0 & 0 \\
\hline III & 19 & 0 & - & 0 & 0 & 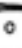 & 0 & $\sigma$ & $>$ & 0 \\
\hline IV & 23 & $\circ$ & 0 & 0 & 0 & $\circ$ & $\circ$ & 7 & 0 & 0 \\
\hline s & 20 & 0 & 0 & 0 & & $\bar{\gamma}$ & $\overline{0}$ & 。 & 0 & $\circ$ \\
\hline
\end{tabular}

Especialidad: Educación Especial

Número de alumnos : 22

P E R F I L

\begin{tabular}{|c|c|c|c|c|c|c|c|c|}
\hline \multirow{2}{*}{ Escale } & \multirow{2}{*}{ P. D. } & \multirow{2}{*}{$\begin{array}{c}\mathrm{Mal} \\
1\end{array}$} & No sstisfictorio & \multicolumn{2}{|c|}{ Normal } & \multicolumn{2}{|c|}{ Bien } & \multirow{2}{*}{$\begin{array}{c}\text { Excelente } \\
9\end{array}$} \\
\hline & & & 23 & 45 & 6 & 7 & 8 & \\
\hline I & 20 & 0 & 0 & 0 & $\circ$ & 0 & $\circ$ & 0 \\
\hline II & 9 & 0 & $\longrightarrow$ & 0 & 0 & 0 & 0 & 0 \\
\hline III & 18 & 0 & 0 & $\circ$ & o & & & 0 \\
\hline IV & 21 & 0 & 0 & 0 & & 0 & 0 & 0 \\
\hline $\mathbf{s}$ & 20 & 0 & 0 & 2 & 0 & 0 & 0 & 0 \\
\hline
\end{tabular}

Especialidad: Lengua Extranjera

Número de alumnos : 12

P E R F I L

\begin{tabular}{|c|c|c|c|c|c|c|c|c|c|c|}
\hline \multirow{2}{*}{ Escals } & \multirow{2}{*}{ P. D. } & \multirow{2}{*}{$\begin{array}{c}\mathrm{Mal} \\
1\end{array}$} & \multicolumn{2}{|c|}{ No satisfactorio } & \multicolumn{3}{|c|}{ Normal } & \multicolumn{2}{|c|}{ Bien } & \multirow{2}{*}{$\begin{array}{c}\text { Excelente } \\
9\end{array}$} \\
\hline & & & 2 & 3 & 4 & 5 & 6 & 7 & 8 & \\
\hline I & 22 & 0 & 0 & 0 & 0 & 0 & 0 & $\rightarrow$ & $\circ$ & 0 \\
\hline II & 11 & 0 & 0 & 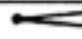 & 0 & $\circ$ & 0 & 0 & $\circ$ & 0 \\
\hline III & 20 & 0 & 0 & 0 & 0 & 0 & 0 & & م & $\circ$ \\
\hline IV & 21 & 0 & 0 & 0 & 0 & 0 & & 0 & 0 & $\circ$ \\
\hline s & 20 & 0 & 0 & 0 & & 0 & $\mathbf{0}$ & 0 & 0 & o \\
\hline
\end{tabular}

Especialidad: Educación infantil

Número de alumnos : 29

P E R F I L

\begin{tabular}{|c|c|c|c|c|c|c|c|c|c|c|}
\hline \multirow{2}{*}{ Escala } & \multirow{2}{*}{ P. D. } & \multirow{2}{*}{$\begin{array}{c}\text { Mal } \\
1\end{array}$} & \multicolumn{2}{|c|}{ No satisfactorio } & \multicolumn{3}{|c|}{ Normal } & \multicolumn{2}{|c|}{ Bien } & \multirow{2}{*}{$\begin{array}{c}\text { Excelente } \\
9\end{array}$} \\
\hline & & & 2 & 3 & 4 & 5 & 6 & 7 & 8 & \\
\hline I & 22 & 0 & 0 & 0 & 0 & 0 & 0 & $\bullet$ & 0 & $\circ$ \\
\hline II & 12 & 0 & 0 & 0 & & & $\circ$ & 0 & 0 & 0 \\
\hline III & 20 & 0 & 0 & 0 & o & o & o & & $>$ & $\circ$ \\
\hline IV & 21 & 0 & 0 & 0 & 0 & & & o & 0 & 0 \\
\hline $\mathrm{s}$ & 20 & 0 & o & 0 & & 0 & 0 & 0 & 0 & 0 \\
\hline
\end{tabular}


Especialidad: Educación Física

Número de alumnos : 10

P E R F I L

\begin{tabular}{|c|c|c|c|c|c|c|c|c|c|c|}
\hline \multirow{2}{*}{ Escals } & \multirow{2}{*}{ P.D. } & \multirow{2}{*}{$\begin{array}{c}\mathrm{Mal} \\
1\end{array}$} & \multicolumn{2}{|c|}{ No sotisfactorio } & \multicolumn{3}{|c|}{ Normel } & \multicolumn{2}{|c|}{ Bien } & \multirow{2}{*}{$\begin{array}{c}\text { Excolente } \\
9\end{array}$} \\
\hline & & & 2 & 3 & 4 & 5 & 6 & 7 & 8 & \\
\hline I & 20 & $\circ$ & 0 & 0 & 0 & & 0 & 0 & 0 & $\circ$ \\
\hline II & 9 & $\circ$ & $\leftarrow$ & & $\circ$ & $\circ$ & $\circ$ & 0 & o & 0 \\
\hline III & 18 & 0 & 0 & 0 & $\circ$ & 。 & o & 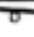 & - & 0 \\
\hline IV & 23 & 0 & 。 & 。 & $\circ$ & - & o & 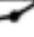 & 。 & - \\
\hline $\mathrm{s}$ & 21 & - & 0 & - & $\circ$ & - & o & 0 & 。 & 0 \\
\hline
\end{tabular}

Especialidad: Educación Primaria

Número de alumnos : 23

P E R F I L

\begin{tabular}{|c|c|c|c|c|c|c|c|c|c|c|}
\hline \multirow{2}{*}{ Escals } & \multirow{2}{*}{ P. D. } & \multirow{2}{*}{$\begin{array}{c}\text { Mal } \\
1\end{array}$} & \multicolumn{2}{|c|}{ No salisfactorio } & \multicolumn{3}{|c|}{ Normal } & \multicolumn{2}{|c|}{ Bien } & \multirow{2}{*}{$\begin{array}{c}\text { Exceiente } \\
9\end{array}$} \\
\hline & & & 2 & 3 & 4 & 5 & 6 & 7 & 8 & \\
\hline I & 19 & 0 & 0 & 0 & 0 & & 0 & 0 & o & 0 \\
\hline III & 8 & 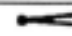 & 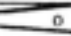 & $\circ$ & 0 & 0 & 0 & $\circ$ & $0^{\prime}$ & 0 \\
\hline III & 17 & - & $\circ$ & 0 & $\circ$ & - & 8 & 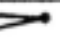 & 。 & 0 \\
\hline IV & 19 & 。 & 0 & 0 & $\overline{0}$ & F & $\%$ & 0 & 0 & 0 \\
\hline$s$ & 21 & 0 & 0 & 0 & 0 & $t$ & 0 & 0 & 0 & 0 \\
\hline
\end{tabular}

Especialidad: Educación Social

Número de alumnos: 19

P E R F I L

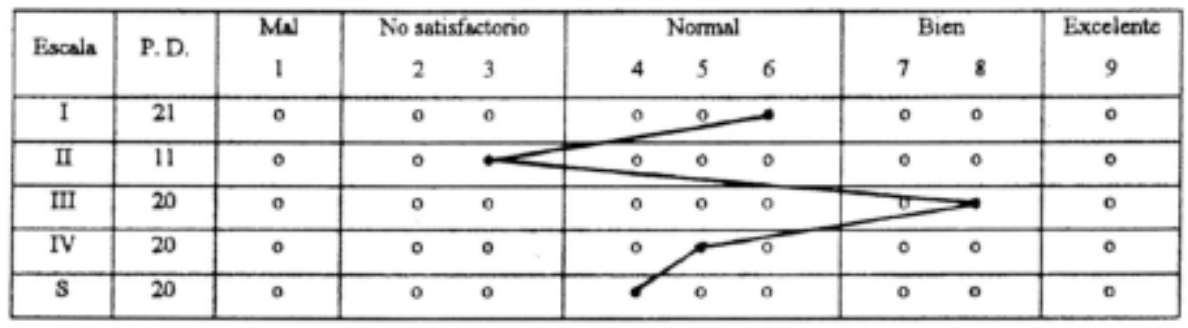

Especialidad: Todas (Magisterio y Educación social)

Número de alumnos : 137

P E R F I L

\begin{tabular}{|c|c|c|c|c|c|c|c|c|}
\hline \multirow{2}{*}{ Escala } & \multirow{2}{*}{ P. D. } & \multirow{2}{*}{$\begin{array}{c}\mathrm{Mal} \\
1\end{array}$} & No satisfactorio & \multicolumn{2}{|c|}{ Normal } & \multicolumn{2}{|c|}{ Bien } & \multirow{2}{*}{$\begin{array}{c}\text { Excelente } \\
9\end{array}$} \\
\hline & & & 3 & 4 & 6 & 7 & 8 & \\
\hline I & 21 & 0 & 0 & $\circ$ & e. & 0 & 0 & $\circ$ \\
\hline II & 10 & 0 & $m=1$ & $\circ$ & 0 & 0 & 0 & o \\
\hline III & 19 & 0 & 0 & 0 & $\circ$ & & & 0 \\
\hline IV & 21 & 0 & 0 & 0 & 0 & 0 & 0 & 0 \\
\hline $\mathrm{s}$ & 20 & 0 & 0 & & 0 & 0 & 0 & 0 \\
\hline
\end{tabular}

\section{4.- Interpretación de los resultados}

De la observación de los perfiles se advierte con facilidad que hay una gran similitud en los hábitos de estudio de las distintas especialidades. Salvo pequeñas diferencias en alguna de las escalas del inventario, todas las submuestras de alumnos obtienen puntuaciones más bajas en la escala correspondiente a la planificación del estudio, es decir, en todo lo que se refiere a la confección de horarios y a la organización del trabajo académico. De hecho, con la excepción de la especialidad de educación 
infantil, todas las gráficas reflejan un nivel insatisfactorio en planificación. En el caso de educación primaria las deficiencias detectadas en esta dimensión son extremas.

Otro hecho destacable es que ninguna de las especialidades obtiene puntuaciones muy elevadas en ninguna de las escalas del inventario. Según se aprecia en las tablas no se alcanza la categoría de excelente en ningún caso.

\section{CONCLUSIONES}

Los resultados obtenidos en la investigación, antes mostrados, nos proporcionan mucha información sobre los hábitos de estudio de los futuros educadores. Si conocer el proceso de aprendizaje de los universitarios es de por sí importante, en nuestro caso esta labor adquiere un valor aún mayor, por tratarse de personas que en un período relativamente breve deberán enseñar a estudiar a sus alumnos.

Numerosos trabajos (Pozar 1989; Salas 1990; Hernández y García 1991; Martínez-Otero 1997) enfatizan la importancia de los hábitos de estudio y su incidencia en el rendimiento académico. En el caso de los alumnos de Magisterio y de Educación Social estos hábitos tienen más trascendencia, porque deberán favorecer en un futuro próximo el aprendizaje de otras personas.

La investigación realizada se halla en su primera fase: necesidad de intervención y valoración inicial, ya que se orienta a conocer los hábitos de estudio de los alumnos de Magisterio y de Educación Social. Tras esta recogida de información y una vez identificado el "problema", nos proponemos diseñar un plan de actuación que permita corregir la negativa situación de partida. El programa de intervención psicopedagógica se encamina a que los alumnos adquieran y consoliden hábitos de estudio que les ayuden a mejorar su rendimiento académico, al tiempo que se forman íntegramente. Así pues, las acciones tendentes a mejorar el estudio de los alumnos no sólo se centran en la enseñanza de técnicas, sino también en la promoción de actitudes y valores positivos. De igual modo, se parte de las necesidades, intereses y características de estos alumnos, lo que equivale a tener en cuenta las relevantes funciones que deberán realizar, los perfiles profesionales de las distintas especialidades y su común denominador como educadores del siglo XXI.

\section{BIBLIOGRAFÍA}

HeRnandeZ, Pedro y Garcia, Luis. Psicología y enseñanza del estudio, Madrid, Pirámide, 1991.

MARTINEZ-OTERO, Valentín. Los adolescentes ante el estudio. Causas y consecuencias del rendimiento académico, Madrid, Fundamentos, 1997.

POZAR, Francisco Inventario de hábitos de estudio (I.H.E.), Madrid, TEA, 1987.

SALAS, Miguel. Técnicas de estudio para enseñanzas medias y universidad_Madrid, Alianza Editorial, 1990. 


\title{
Contactar
}

Revista lberoamericana de Educación

\author{
Principal OEI
}

